\title{
A Novel Minimally Invasive Retro Rectus Repair of Ventral Hernia
}

Comparing Kumar-Subhash's modified eTEP technique with Laparoscopic Intraperitoneal Onlay Mesh Hernioplasty

\section{Background}

Ventral hernia repair is one of the common general surgical procedures. A novel eTEP technique has been developed where in a mesh is placed in retro muscular plane through minimally invasive approach. There is scarcity of data on its effectiveness as compared to conventional Laparoscopic IPOM hernioplasty.

\section{Aims and Objectives}

We aim to introduce our innovative Kumar-Subhash's modified 3 port eTEP technique for ventral hernia repair and compare its clinical effectiveness with conventional IPOM repair.

\section{Methods}

A prospective comparative study was undertaken at Devagiri Hospital, Bangalore, India from January 2017 to December 2019. 30 patients were included in the study (12 in eTEP group and 18 in IPOM group) based on predefined inclusion and exclusion criteria. Patients were followed up to 30 days post-operatively. The outcomes were compared with respect to postoperative pain, length of hospital stay and early postoperative complications.

\section{Results}

Baseline characteristics and presenting complaints were comparable for both the groups. The mean pain score on POD 1 in eTEP group was 3 , and 1 on POD 7 as compared to 7 and 3 in IPOM group on POD1 and 7 respectively, which was statistically significant. Patients in ETEP group had a shorter length of hospital stay (LOS). None of the patients had any complications related to the novel technique.

\section{Conclusions}

Kumar-Subhash's modified 3 port eTEP technique is a novel and easy approach for laparoscopic repair of ventral hernia with significantly less post-operative pain and LOS with an additional cosmetic advantage for patients.

Keywords: eTEP, IPOM, ventral hernia, Paraumbilical hernia, retrorectus, mesh.
Anil Kumar ${ }^{1}$ (D) Meghana Taggarsi ${ }^{2}$ (i) \& R.C. Subhash ${ }^{3}$

1 Department of General and Colorectal Surgery, Stockport NHS Foundation Trust, Stepping Hill Hospital, Stockport, UK; 2 Department of General and HPB Surgery, Royal Blackburn Hospital, Blackburn, UK; 3 Department of General and Minimally Invasive Surgery, Devagiri Hospital, Bengaluru, India

Correspondence to nettyanil@gmail.com

Cite as; Kumar, A., Taggarsi, M. \& Subhash, R.C. (2021) A novel minimally invasive retro rectus repair of ventral hernia - comparing Kumar Subhash's modified e-TEP technique with laparoscopic intraperitoneal onlay mesh hernioplasty. The Physician vol 7; Issue 1: ePub 4 Aug'21 DOI https://doi.org/10.38192/1.7.1.11

$\underline{\text { Article Information }}$

Submitted 8.7.21

Published 4.8.21

ISSN 2732-513X (Print) ISSN 2732-5148 (Online)

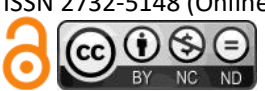

scienceOPEN.com

Introduction: 
Ventral hernia repair is one of the common general surgical procedures. They were done by open approaches until 1993. It was in 1993 that LeBlanc and Booth described laparoscopic repair of ventral hernia for the first time [1]. Till then, laparoscopic approach was limited to repair of inguinal hernia which included Totally extraperitoneal repair (TEP), Transabdominal preperitoneal repair (TAPP) and intraperitoneal onlay repair. There has been a constant search for innovative procedures in the field of abdominal wall hernias. Conventional methods like open onlay mesh hernioplasty, open retro muscular mesh hernioplasty (Rives-Stoppa) and laparoscopic intraperitoneal onlay mesh (IPOM) hernioplasty are popular [2].

Evidence suggests that retro muscular mesh hernioplasty has advantages over other techniques as far as recurrence is concerned [2]. An alternative technique has been developed where a mesh is placed in retro muscular plane by minimal access technique extended totally extraperitoneal repair (eTEP). This was first introduced by Jorge Daes in 2012 to address difficult inguinal hernias, particularly the issue with operating in a limited space while performing TEP [3]. Following which, Dr. Yuri Novitsky, Dr. Igor Belyansky described the eTEP technique to repair ventral hernias. Their multicentre study proved its efficacy and feasibility in ventral hernias [4]. The eTEP retro-rectus technique is based on the same principles and technical steps as used by the Rives-Stoppa open repair. The concept and technique of eTEP for repair of ventral hernia is very novel and there is worldwide scarcity of data on its application for treatment of ventral hernias.

The literature is sparse with regards to prospective studies done to compare the effectiveness of eTEP technique with the conventional laparoscopic IPOM repair for ventral Hernia and hence, we decided to conduct a study to look into this aspect and compare the early post-operative outcomes of the two techniques.

\section{Aims and Objectives:}

We conducted a prospective single centre study to compare the effectiveness of modified eTEP technique with that of the conventional IPOM repair for ventral hernia in terms of early postoperative outcomes. The study also aims to introduce the technical aspects of our modification of originally described eTEP technique, which we intend to name after the surgeons involved, Kumar-Subhash's Modified e-TEP technique.

\section{Materials and Methods:}

We conducted a prospective comparative study from January 2017 to December 2019 at Devagiri Hospital, Bangalore, India. 30 patients were included in the study.
The ethical approval was obtained by the Institutional Review Board. The inclusion criteria comprised of patients aged 18 and above, presenting with epigastric, umbilical or paraumbilical hernia, with a defect size of 2 $-3 \mathrm{cms}$, and having no contraindication for laparoscopic surgery, while patients with multiple defects, poor skin condition overlying the hernia, recurrent hernia, hernia with strangulation or obstruction, previous open upper abdominal surgeries like laparotomy, and previous hernia with divarication of recti were excluded from the study. The mesh used in all the patients was standardised.

A total of 30 patients who met the inclusion criteria were included in the study. Patients were divided into two groups, group 1 comprised of 12 patients who underwent laparoscopic eTEP and group 2 included 18 patients who underwent conventional laparoscopic IPOM repair. All the patients underwent repair of primary ventral hernia. The allocation of patients to the eTEP arm or IPOM arm depended on patient's choice. Since, we were adopting a new technique, both the procedures were explained to patient and their choice was respected. Informed consent was obtained from all the patients included in the study. The post-operative data was entered by an independent clinical staff who was blinded from the type of surgery the patient underwent. The postoperative outcomes were compared with respect to postoperative pain, duration of hospital stay and early post-operative complications which included wound infection, mesh infection, seroma or hematoma. Postoperative pain was assessed using VAS (Visual Analogue score) on day 1 and day 7 of surgery. All the patients in both groups were provided with a standard post-operative pain medication which comprised of intravenous/oral paracetamol. All the patients were followed up for a period of 30 days postoperatively. (Figure 1)

\section{Technique of Kumar-Subhash's Modified eTEP procedure (Figure 2):}

Our port placement and dissection were different from the one described by $\mathrm{Dr}$ Belyansky et al. The port placement and technique followed were same in all patients who underwent eTEP. Our method involves securing 3 ports instead of originally described 4 port technique.

Port placement and access to retro-rectus plane (Figure $2 a, 2 b, 2 c)$ :

After induction of general anaesthesia, with patient in supine position, incision for the first port was made in left hypochondriac region in the mid clavicular line. The anterior rectus sheath was identified and divided. The 
rectus abdominis muscle was retracted or split to access the retro-rectus space, $10 \mathrm{~mm}$ primary port was inserted and pneumo was created. Initial plane creation was done using the 0 -degree laparoscope which can later be changed to 30-degree scope depending upon surgeon's convenience and then a $5 \mathrm{~mm}$ left lumbar port was placed under vision to aid the appropriate plane creation. Similarly, another $10 \mathrm{~mm}$ port was placed in right hypochondriac region to help in the creation of retro-rectus plane on the right side.

Crossing Over midline (Figure 2d, 2e):

The medial edge of left posterior rectus sheath was identified and divided and the pre peritoneal plane below the linea alba was identified. Similarly, the medial edge of contralateral right posterior rectus sheath was identified by instrument placed from right hypochondriac port and divided.

Development of the retro-rectus plane, Dissection of sac and placement of Mesh (Figure $2 f, 2 g, 2 h$ ):

After cross over, telescope was shifted to right hypochondriac port. Dissection was continued using left lumbar and left hypochondriac ports, to create adequate retro-rectus space, cephalad to caudal and the lateral limit of dissection was limited to the medial margin of linea semilunaris. The contents of sac were reduced into peritoneal cavity and adhesions released. A $15 \times 15 \mathrm{cms}$ polypropylene mesh was placed in retrorectus plane \& anchored using 2 midline transfascial sutures. No tackers were used. Pneumo was released under vision assuring that the mesh is lying flat between the posterior and anterior rectus sheath layers. A pressure dressing was applied over the defect area for a period of 48-72 hours to prevent development of seroma/haematoma.

\section{Technique of IPOM:}

A standard 3 port technique was used to access peritoneal cavity. After adhesiolysis and reduction of the hernial contents, a $15 \times 15 \mathrm{cms}$ composite mesh was used. 4 non-absorbable tackers and 4 absorbable transfascial sutures were used to secure the mesh to peritoneum.

In both the techniques, the defect was not closed, and sac was not sutured.

\section{Statistical methods:}

Statistical Analysis was done using SPSS version 25. All eligible patients were included in the analysis in the group they were allocated. Descriptive analysis of continuous and categorical variables was done in terms of frequencies and standard deviations. Inter-group analysis was done using Pearson's Chi square test and independent ' $\mathrm{t}$ ' test. $A$ ' $p$ ' value less than 0.05 was considered significant, with $95 \%$ confidence interval. The power of the study was calculated to be more than $90 \%$.

\section{Results:}

In our study, total of 30 patients were included, who met the set inclusion and exclusion criteria of the study. 12 of these patients underwent eTEP and 18 patients underwent laparoscopic IPOM repair. The mean age of patients undergoing ETEP and IPOM repair was 44 years and 48 years, respectively, the $p$ value being 0.681 , not statistically significant. In group 1, 8 of the patients were females and 4 were males. In group 2, 11 patients were females and 7 of them were males. The $p$ value was 0.534.(Table 1)

In group 1, 11 patients underwent eTEP for paraumbilical hernia, while 1 had the surgery for epigastric hernia. In group 2, all the patients underwent the surgery for paraumbilical hernia. 1 of the patients in group 1 had a TEP repair for inguinal hernia previously, and 1 of the patients had a tubectomy before. None of the patients in group 2 had any previous surgeries. (Table 1)

The VAS score for pain for majority of patients $(n=8)$ who underwent eTEP, on postoperative day 1 was 4 , and the rest $(n=4)$ was 2 . The mean pain score on postoperative day 1 in group 1 was 3 , and on postoperative day 7 it was 1 . While in group 2 , the VAS score on day 1 for majority $(n=10)$ was 8 , and for the rest $(n=8)$ was 4 . The mean pain score on $1^{\text {st }}$ postoperative day was 7 , and on $7^{\text {th }}$ postoperative day the score was 3 . The pain score amongst both groups showed a clinically significant difference, in that, the pain was more in patients who underwent laparoscopic IPOM repair. The pain scores at day 1 and day 7 were statistically significant too, with a p value 0.000 for both. (Table 2 ) All the patients who underwent eTEP stayed in hospital postoperatively for 1 to 2 days. The mean length of hospital stay for these patients was 2 days. However, amongst the patients who underwent IPOM, 5 of them stayed in hospital for 4 days, and 5 stayed for 3 days. The mean hospital stay for patients in group 2 was 3 days, which is also more than that observed in group 1 . This difference was statistically significant, with a $p$ value of 0.001.(Table 2)

One of the patients out of 12 who underwent eTEP had ecchymosis postoperatively, which did not require any definitive management. However, since the sac was not sutured in all the 30 patients, minimal seroma was 
observed in all on examination in the follow up clinic on post-operative day 7. However, none of them were recognised by the patient or complained about by them. They were seen only on examination by the clinician, which subsided on their own, all within 30 days postoperatively.

None of the patients in both the groups had a conversion of surgery to open procedure. No other complications were seen intraoperatively or postoperatively, amongst patients in both groups. All the patients included in the study were followed up to a period of 30 days postoperatively. None of the patients complained of pain, seroma, haematoma, wound infection and recurrence at 30 days of follow-up.

\section{Discussion:}

Many surgeons have for long, tried to use the retrorectus space for hernia repair, with minimal access. With increasing studies and evidence, which suggest better results pertaining to the outcomes, more surgeons are attempting to practice eTEP for ventral hernias. The technique of eTEP was devised by Dr Jorge Daes in 2012 for managing difficult inguinal hernias [3].

We wanted to explore this technique in repairing ventral hernias and devised a modified eTEP technique for the same in later half of 2016 based on the principle of open Rives-Stoppas repair for ventral hernias. The first case using the novel technique was done in 2017, we had excellent postoperative outcomes and subsequently started using the technique more frequently. The technique of eTEP for ventral hernias was later published in 2018 by Dr. Belyansky et al [4]. It is a novel technique that involves creating the retro-rectus space bilaterally and connecting them, using minimal access method. It also involves placing larger meshes sandwiched between the two layers of rectus sheath to cover larger areas. Our technique differs from that of $\mathrm{Dr}$ Belyansky. The Kumar-Subhash's modified e-TEP repair of ventral hernia method involves plane creation using 3 port technique instead of the originally described 4 port technique. Since the mesh is placed in the retro-rectus space, it prevents the risks arising from the direct contact between mesh and intraperitoneal organs especially bowels like mesh erosion, postoperative ileus, adhesions and bowel obstruction.

In our study, the two groups were comparable with respect to age, gender, and presenting complaints. In our study, there was clinically, and statistically significant difference observed in the pain score at postoperative day 1 and day 7, and also a statistically significant difference was observed in the length of hospital stay. A possible explanation of higher pain levels in IPOM group could be due to use of tackers and transfascial sutures used to fix the mesh. As both eTEP and IPOM are laparoscopic methods for repair of ventral hernias, there was no additional cost incurred to the patients per se with respect to the novel technique. Our modified eTEP technique does not involve use of composite mesh and tackers for fixation, hence there may be a potential for reducing the overall cost of minimally invasive ventral hernia repair.

The results of our study are in conjunction with that of Dr Belyansky's. The mean hospital stay in their study was 1.8 days [4]. We did not encounter wound-related complications in any of our patients at the 30 postoperative days.

Issue of chronic pain and recurrence has long been an issue with ventral hernia repairs. Over the years as the method of repairs evolved the incidences of both chronic pain and recurrences have significantly decreased. However, a recent study on IPOM still has reported an occurrence of chronic pain in 3.3 to $7 \%$ of patients $[5,6]$. Reported incidence of prolonged ileus in IPOM is $3 \%$. In our study, patients who underwent eTEP had their pain subsided during the 30 day follow up. None of them had readmissions and ileus during the 30day post-operative period.

A case series reported recently by Shakya et al, included 3 patients who underwent eTEP for recurrent ventral hernias, showed satisfying short-term results [7]. A retrospective study in 2018, conducted a retrospective study comparing ETEP and IPOM. Their results were in conjunction with our study. The pain score (VAS) reduced to the 7th postoperative day, but the intraoperative time was significantly more in eTEP than in IPOM [8]. Although, our study did not look into the intraoperative time.

A retrospective analysis done in 2019, on 24 patients which included inguinal and ventral hernias, reported lesser VAS scores compared to our study. However, the lesser scores were seen in patients who underwent eTEP for inguinal hernias as compared to that for ventral hernias [9].

\section{Limitations}

The literature is sparse with regards to prospective comparative study between the two techniques; however, our study had limitations of a small sample size, involved early postoperative outcomes and had a limited duration follow-up of 30 days. Future randomised studies with large sample size and longterm follow-up would be needed for substantial results 
on this novel technique. Since composite mesh and tackers are not needed for modified eTEP technique, there is good potential for cost saving with this procedure, although cost analysis was not the aim of the study, hence, this was not investigated. There is scope for future studies in this regard, to assess cost benefits of the hereby described eTEP technique.

\section{Conclusion:}

In our prospective study, by modifying the existing 4 ports technique to 3 ports technique, we were able to achieve good results. The modified technique made operating easier. The duration of stay as well as postoperative pain were less with eTEP as compared to conventional technique of laparoscopic IPOM. The use of polypropylene mesh and transfacial sutures in eTEP as compared to composite mesh and tackers in IPOM, contributed to reducing costs incurred from the surgery. However, analysing the costs was beyond the aim of this study.

In the present era of Minimally invasive surgery, KumarSubhash's modified 3 port eTEP technique offers an additional skill to the armamentarium of Minimally invasive surgeons which proves to be a feasible and effective option for repair of ventral hernias with good outcomes.

\section{Declarations}

Funding: None

Conflicts of interest/Competing interests: none.

Code availability: None

Ethical Approval: Yes - Local Ethical Committee.

Consent to participate: Yes, Informed consent was obtained from all patients for being included in the study

Consent for publication: Yes

Availability of data and material: Yes, with the Authors Authors' contributions: All the authors contributed substantially in the conception of the study, design, literature search and write-up of the manuscript. The Abstract was presented as Poster at Forum for Interdisciplinary Research in Medicine, Science \& Technology Conference, Moscow 2021. https://doi.org/10.38192/1.6.3.firmst20.ab36.

\section{References:}

1. LeBlanc KA, Booth WV (1993) Laparoscopic repair of incisional abdominal hernias using polytetrafluoroethylene: preliminary findings. Surgical Laparoscopy and Endoscopy 3(1):3941.

2. Baig SJ, Priya P (2018) Extended totally extraperitoneal repair (eTEP) for ventral hernias: Short-term results from a single centre. Minim Access Surg 15(3):198-203.

3. Daes J (2012) The enhanced view- totally extraperitoneal technique for repair of inguinal hernia. Surg Endoscop 26:1187-1189.

4. Belyansky I, Daes J, Radu VG et al (2018) A novel approach using the enhanced-view totally extraperitoneal (eTEP) technique for laparoscopic retromuscular hernia repair. Surg Endoscop 32:1525-1532.

5. Eriksen JR (2011) Pain and convalescence following laparoscopic ventral hernia repair. Dan Med Bull 58(12):B4369.

6. Misiakos EP, Machairas A, Patapis P et al (2008) Laparoscopic ventral hernia repair: Pros and Cons compared with open hernia repair. JSLS 12(2):117-125.

7. Shakya VC, Byanjankar B, Pandit R et al(2019) e-TEP retromuscular repair for recurrent Incisional hernias: Report of three cases. Hindawi Case Reports in Surgery Article ID 1609193.

https://doi.org/10.1155/2019/1609193.

8. Penchev D, Kotashev G, Mutafchiyski V (2019) Endoscopic enhanced-view totally extraperitoneal retromuscular approach for ventral hernia repair. Surgical Endoscopy 33(11):3749-3756.

9. Deshpande RK, Talwar S (2019) A novel laparoscopic approach: (e-TEP) technique in ventral abdominal hernia-our experience. International Journal of Surgery Science 3(4): 22-25.

https://doi.org/10.33545/surgery.2019.v3.i4a. 212.

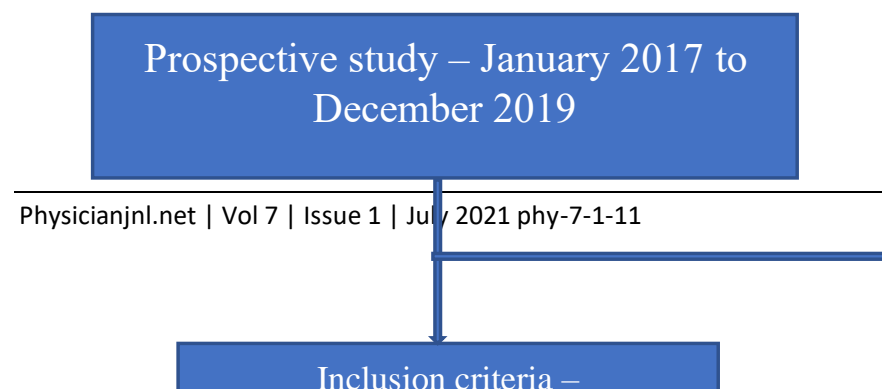

Inclusion criteria -

\section{Exclusion criteria -}

1.Multiple Defects

2.Poor skin condition 
Figure 1. Patient and Methods 


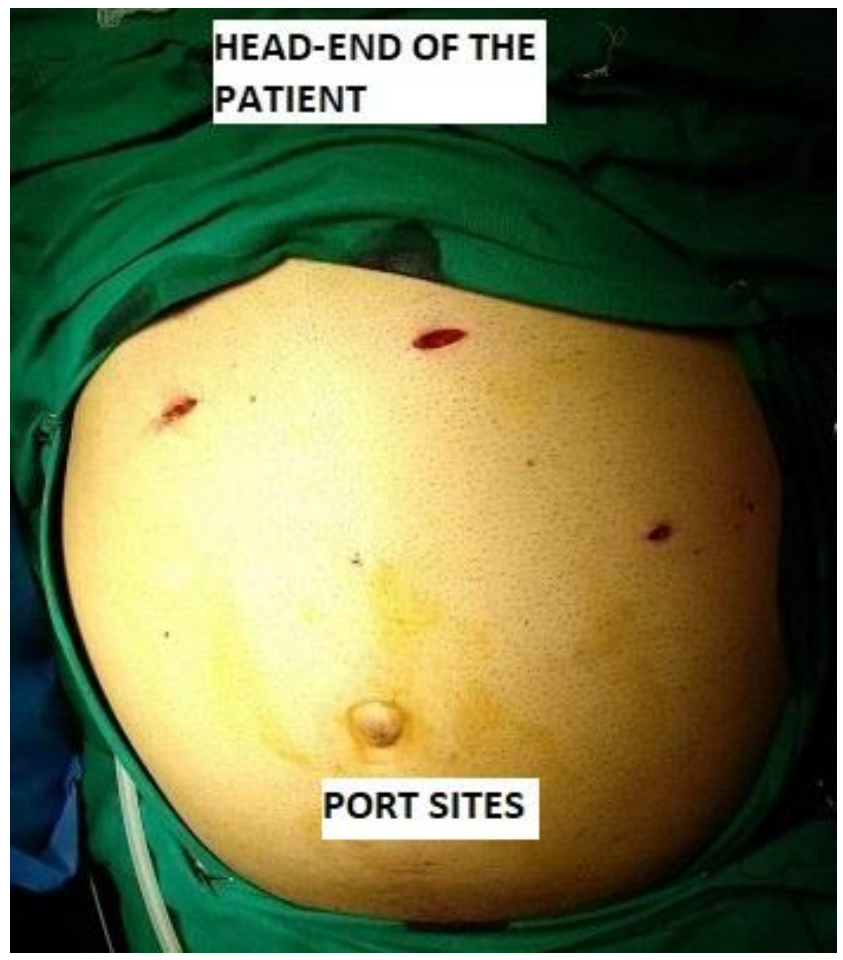

Figure 2a. Port Incision

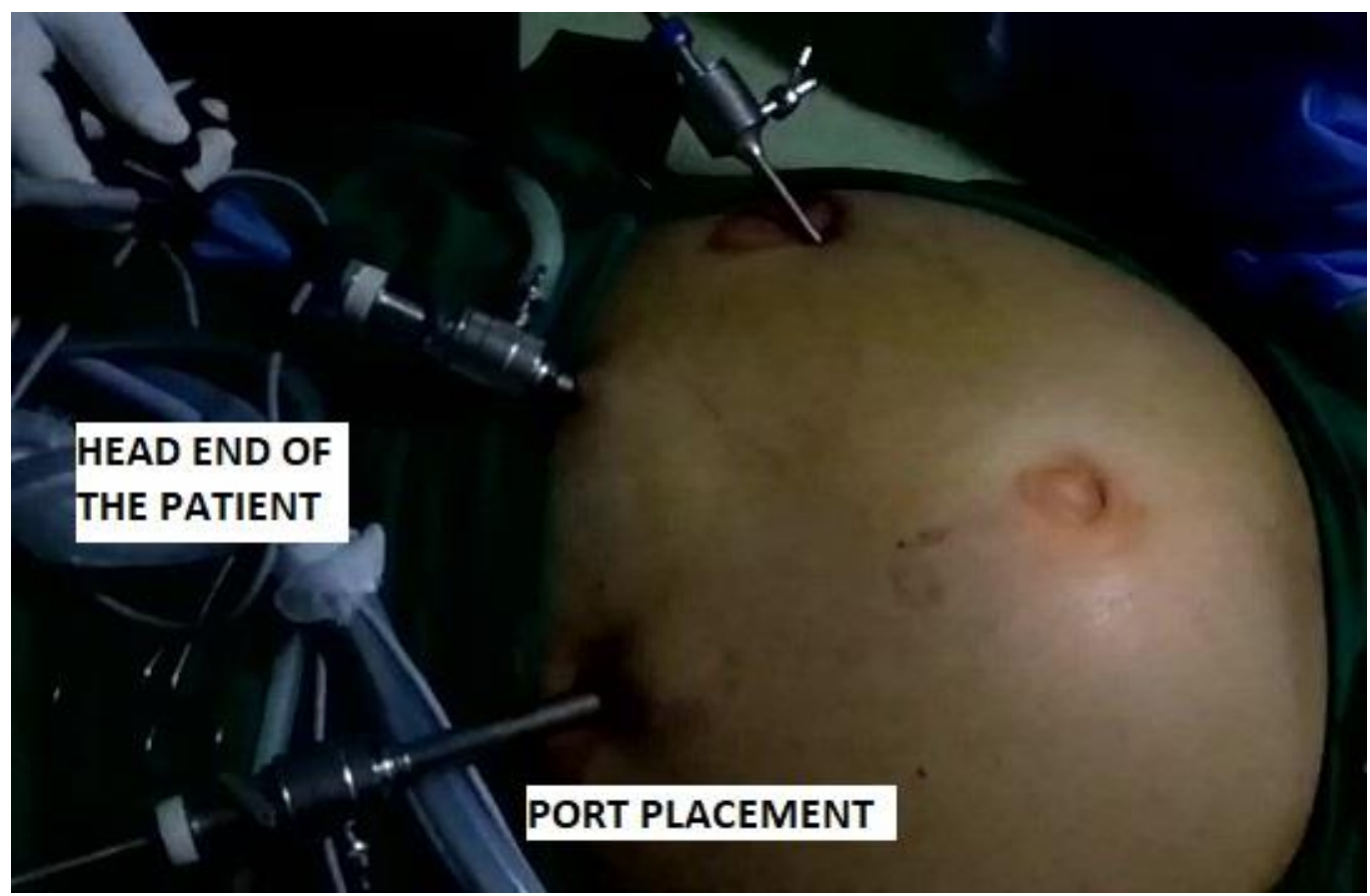

Figure 2b. Ports Placement 


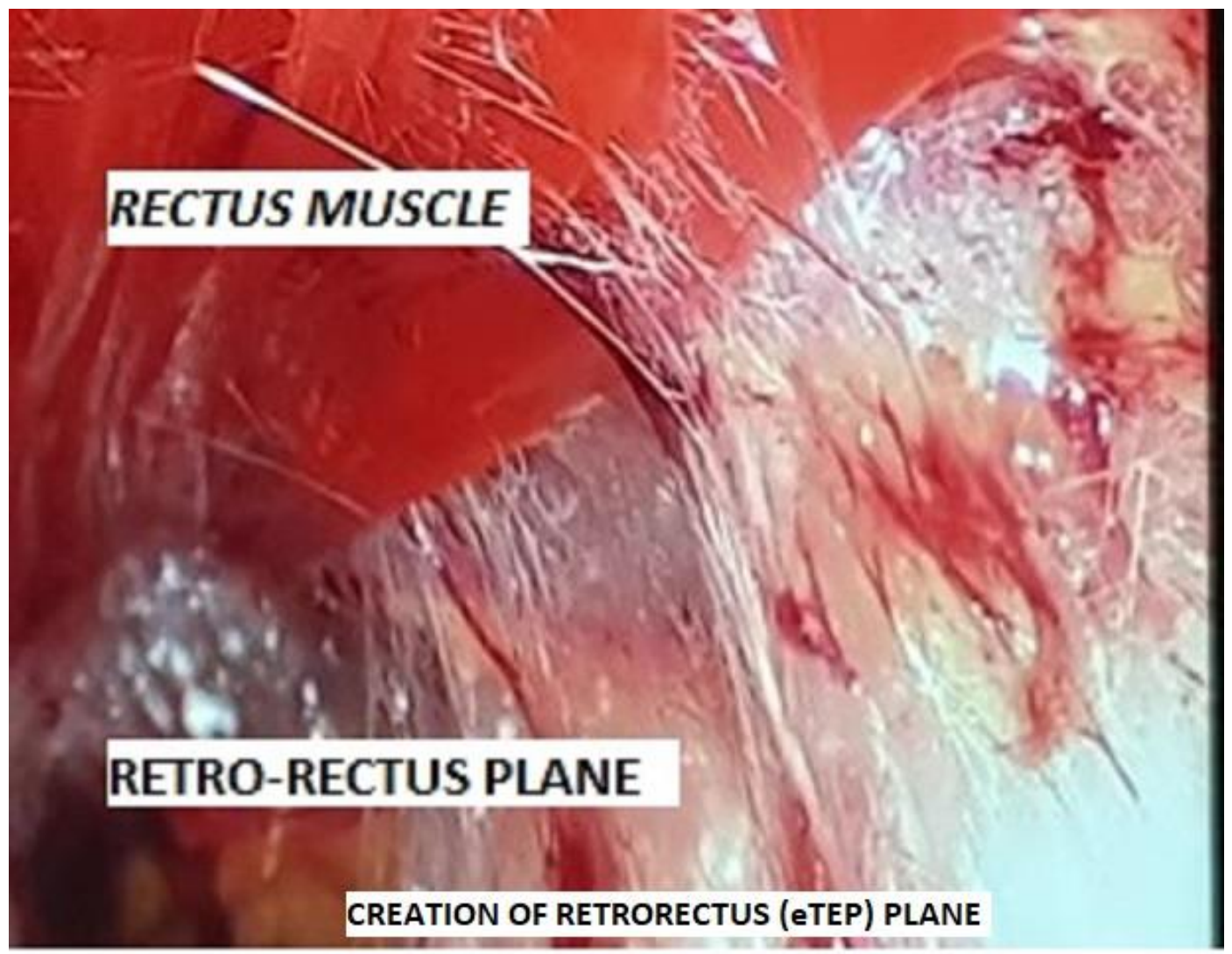

Figure 2c. Creation of Retrorectus plane

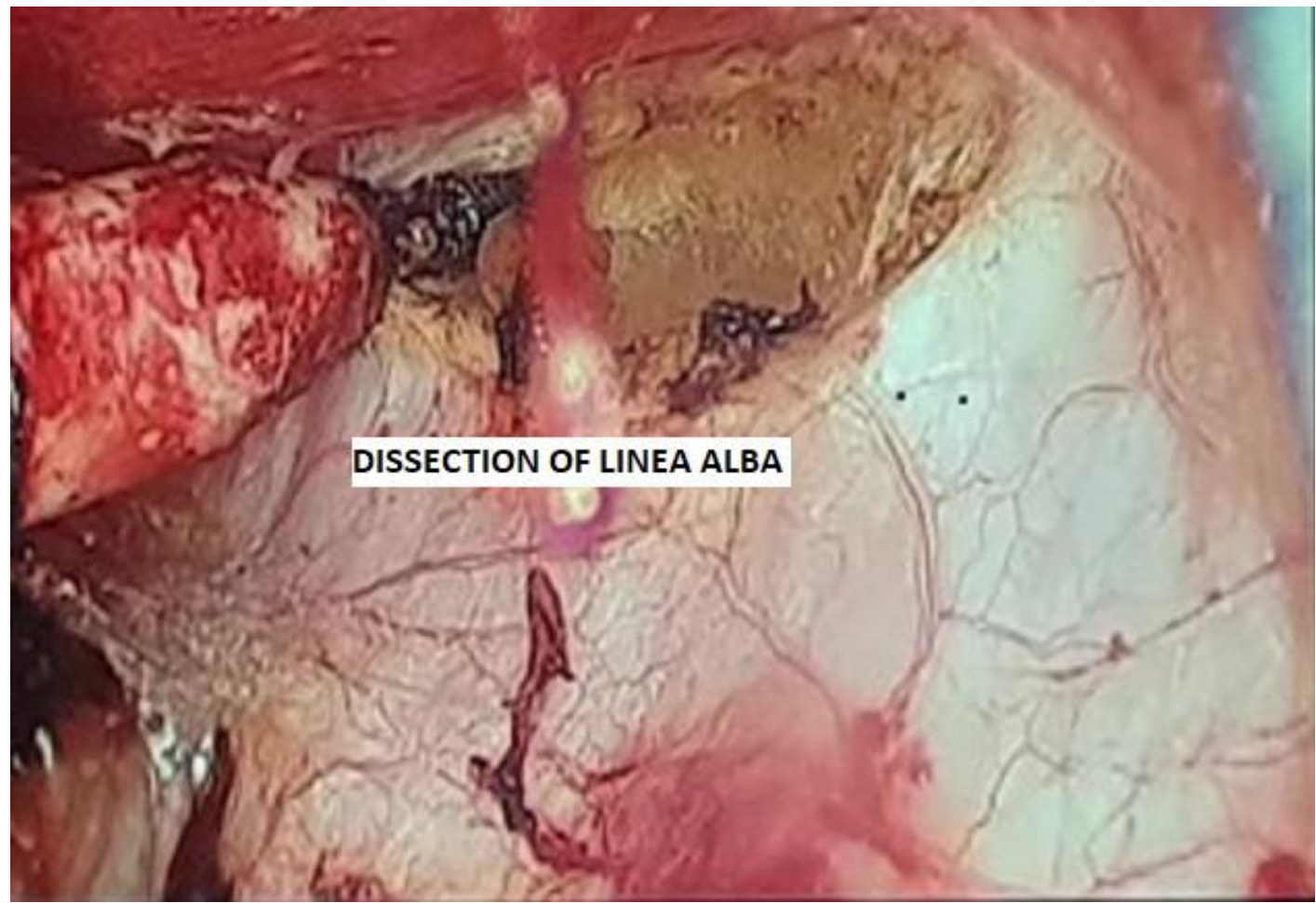

Figure 2d. Dissection of Linea Alba 


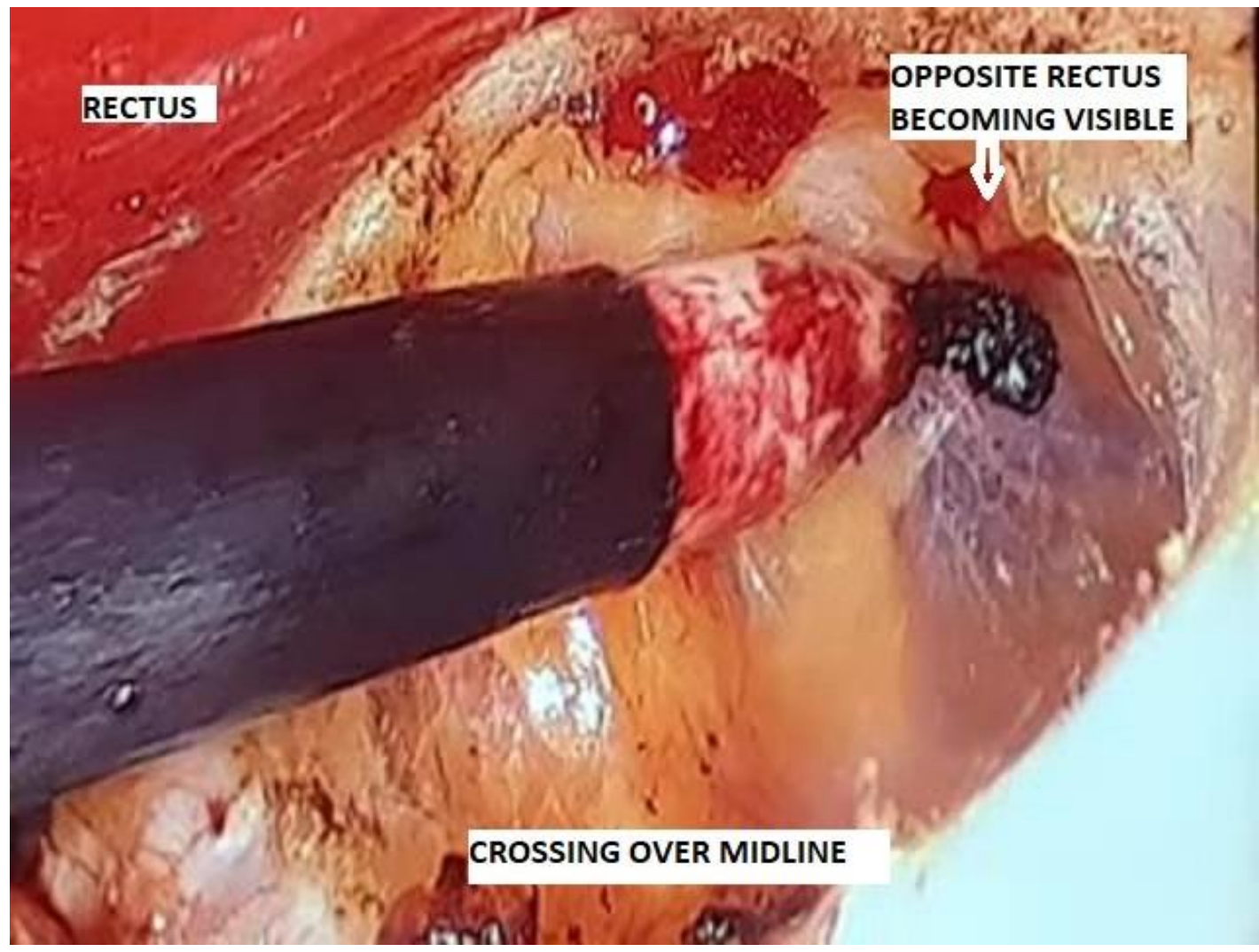

Figure 2e. Crossing over Midline

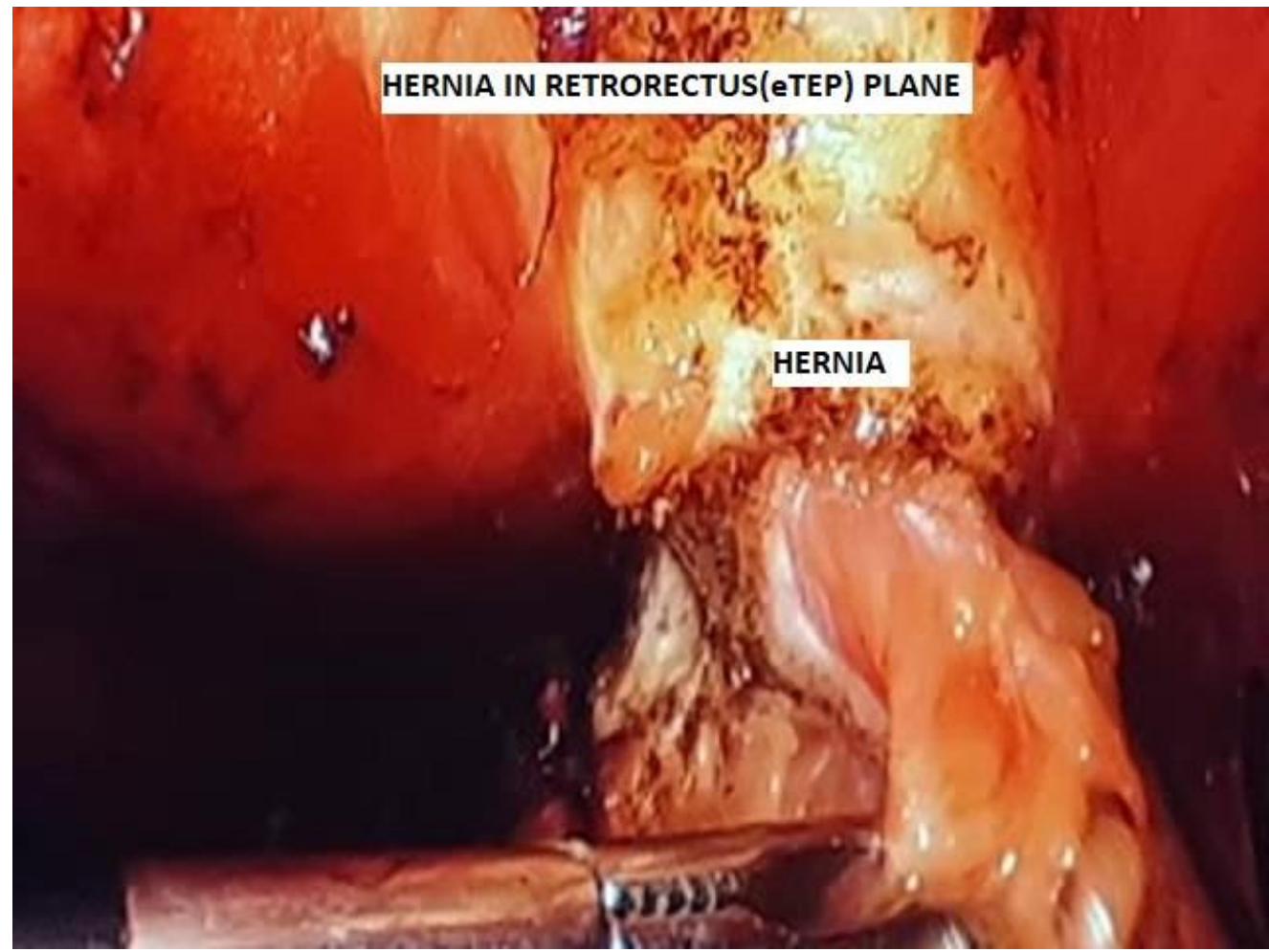

Figure 2f. Hernia in Retrorectus plane 


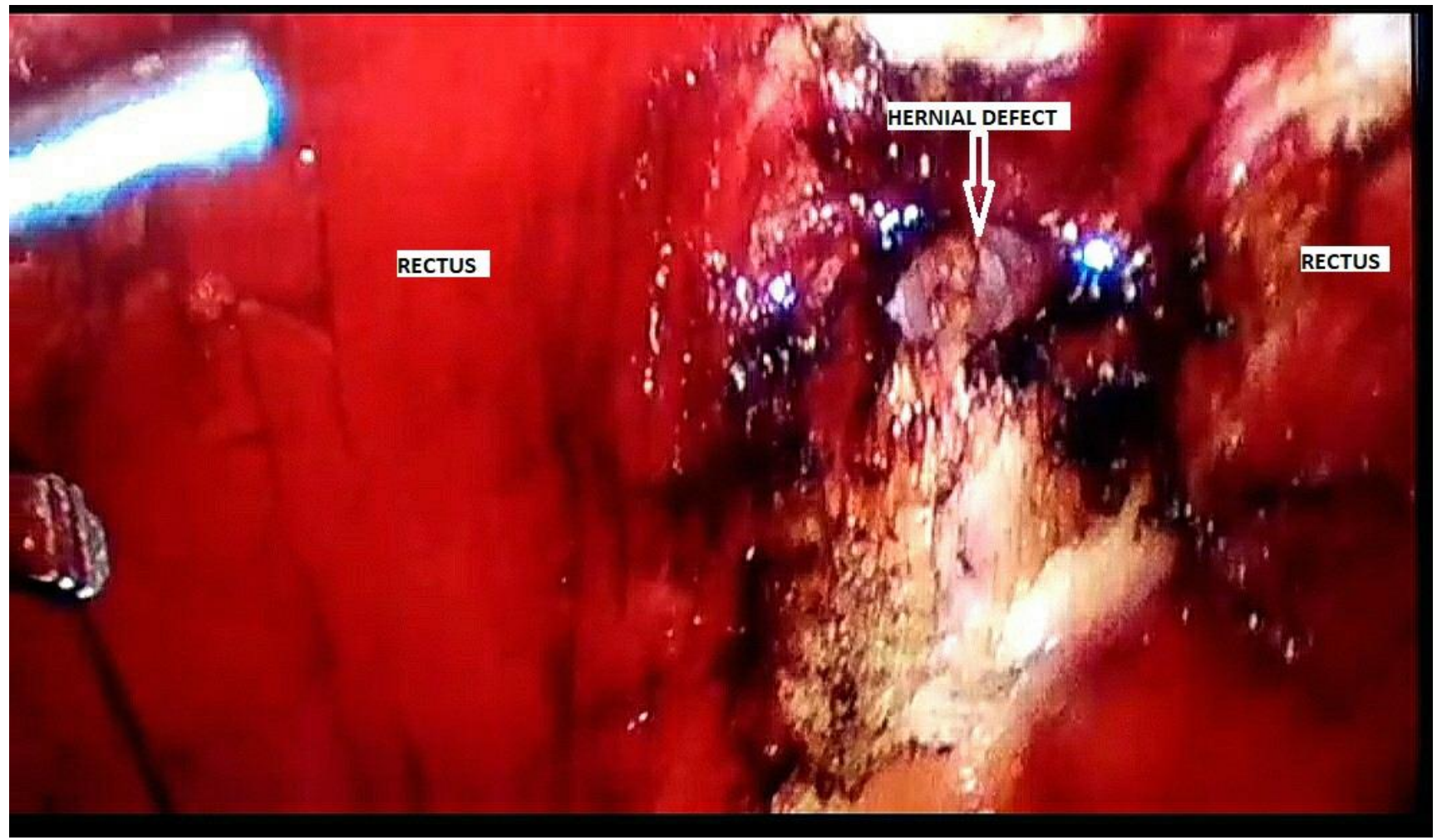

Figure 2g. Hernial Defect

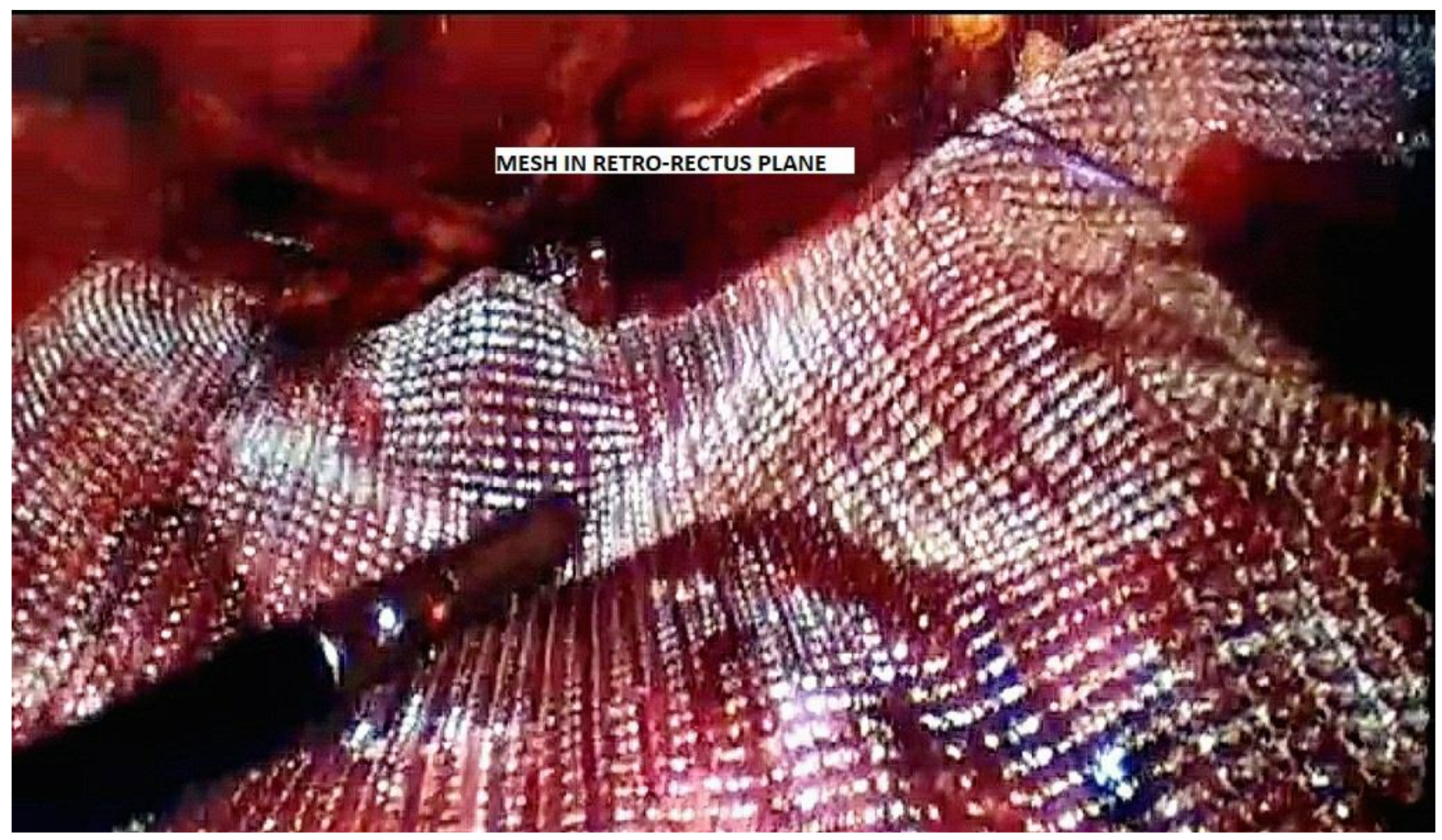

Figure 2h. Mesh in Retrorectus plane

Figure 2. Steps of Kumar-Subhash's Modified eTEP Technique 


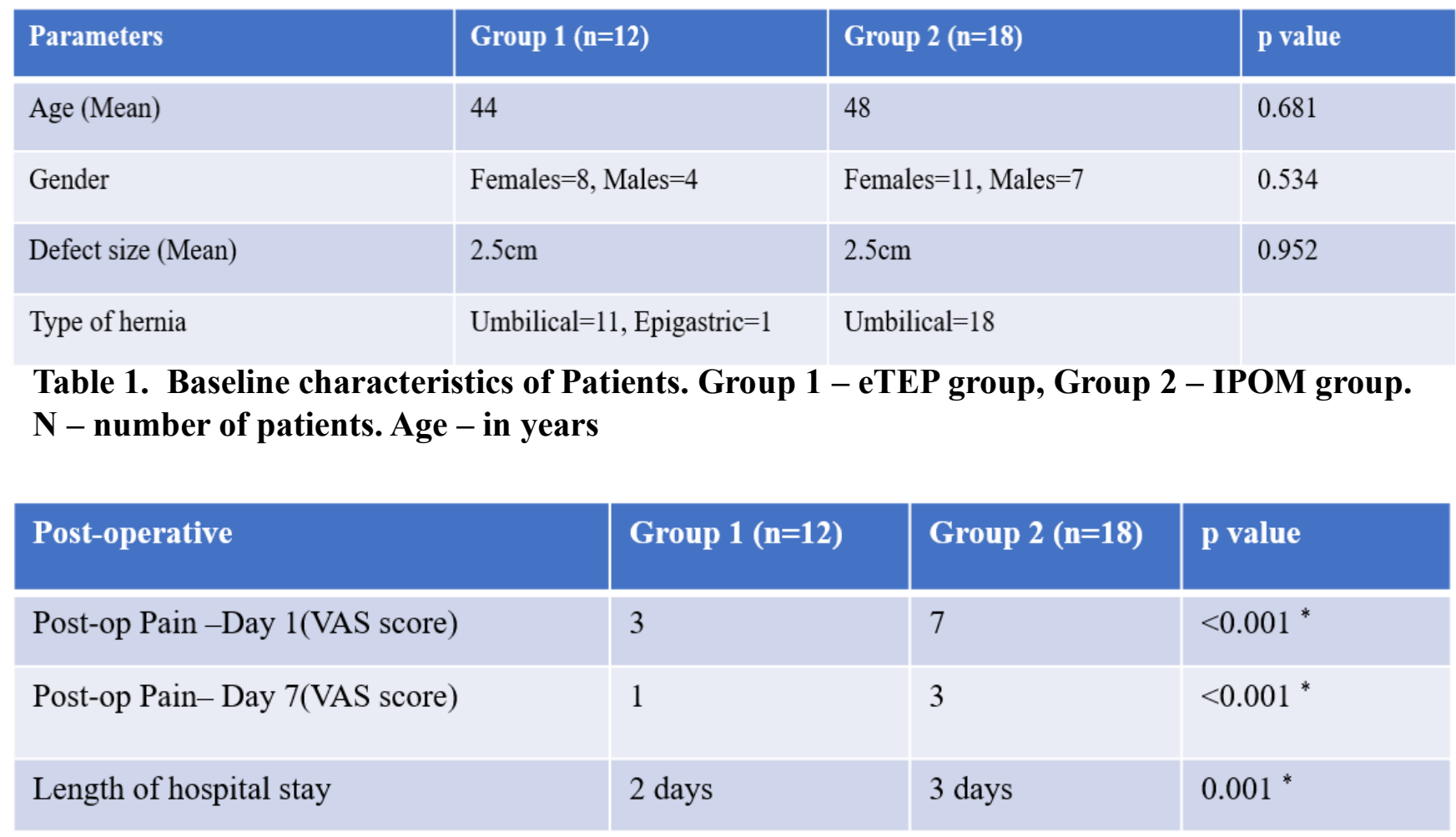

Table 2. Early post-operative Outcomes. * indicates significant p-value. None of the patients had seroma, haematoma, wound infection in the immediate and early post-op period. 